\title{
Measurement of Exciton Diffusion Lengths of Phthalocyanine Derivatives Based on Interlayer Excitation Transfer
}

Musubu Ichikawa ${ }^{1,2, *}$

${ }^{1}$ Department of Bioscience and Textile Techonolgy, Interdisciplinary Graduate School of Science and Technology, Shinshu University, 3-15-1 Tokida, Ueda City, Nagano 386-8567, Japan

${ }^{2}$ Presto, Japan Science and Technology Agency (JST), 4-8-1 Honcho, Kawaguchi, Saitama 332-0012, Japan

(Received )

The exciton diffusion length $\left(L_{\mathrm{D}}\right)$ of several phthalocyanines (Pcs) was investigated by use of organic photovoltaic cells, where two different p-type organic semiconductor materials, including a Pc layer, were laminated, and interlayer excitation energy transfer occurred between them. The distribution of excitons in the Pc layer that accept energy from the other p-type semiconductor material by excitation transfer should be much narrower than that of excitons created by light absorption directly. This delta-function-like generation of excitons allowed the simple analysis of exciton diffusion. The results of the analysis indicated that the planar Pcs (copper Pc and metal-free Pc) showed shorter $L_{\mathrm{D}} \mathrm{S}$ of around $5 \mathrm{~nm}$, as compared to the $18.5 \pm 4.8 \mathrm{~nm}$ 
shown by the pyramidal-shaped Pc (tin Pc). 


\section{Introduction}

Organic photovoltaics (OPVs) have received a lot of attention because of their potential in large-scale and low-cost solar energy conversion $[1,2]$. The generally accepted formation mechanism of an OPV is as follows: (1) absorption of photons by p-type and/or n-type organic semiconducting materials and creation of their excitons, (2) diffusion of excitons to the interface of both materials ( $\mathrm{p} / \mathrm{n}$ junction), (3) dissociation of excitons into electrons and holes at the junction, and (4) collection of the charged carriers at the electrodes. State-of-the-art OPV cells with high power conversion efficiency are based on bulk heterojunction (BHJ) [3] or p-i-n [4] architecture in order to overcome a fundamental issue of organic semiconducting materials, i.e., the short exciton diffusion length $\left(L_{\mathrm{D}}\right)$. In photoactive layers, the heterojunctions required for dissociating an exciton into charged carriers should be located within the $L_{\mathrm{D}}$ of the light-absorbing layers from any part of the photoactive layer. Thus, the BHJ architecture is important; however, in the case of planar heterojunction architecture, the thickness of the light-absorbing layer should be shorter than its $L_{\mathrm{D}}$. Hence the $L_{\mathrm{D}}$ of the semiconductors is a pivotal material property for OPVs.

To date, the reported values of $L_{\mathrm{D}}$ obtained mainly by the photoluminescence quenching technique have ranged widely for various materials [5-10]. Although this technique is arguably most useful for luminescent materials, it cannot be applied to nonluminescent materials. Phthalocyanine $(\mathrm{Pc})$ and its derivatives are widely used as a p-type semiconductor material in OPV, but most of them are not luminescent. The thickness dependence of the photocurrent response in solar cells is another technique to determine $L_{\mathrm{D}}$, but a simple exponential distribution of excitons based on optical penetration depth could be misleading because of the optical interference arising from 
the reflective metal counter electrode [11]. Some literature taking this influence into account has been published, but we believe it is controversial to determine $L_{\mathrm{D}}$ from the thickness dependence of photocurrent response on the basis of reported $L_{\mathrm{D}}$ values that can vary from a few to tens of nanometers; like for example, in the case of copper Pc $(\mathrm{CuPc})[12-17]$

Recently, we demonstrated OPV cells exhibiting a photoenergy collection and transfer system based on interlayer excitation transfer (ET) [18]. The cells have two different p-type organic semiconductor layers, with one layer absorbing photons and transferring its exciton energy to the other layer (from $\mathrm{p} 1$ to $\mathrm{p} 2$ ), as shown in the inset of Fig. 1. Excitons in the ET-accepting p-type layer should be produced very near the $p / p$ interface, unlike in the case of optical excitations. These situations are schematically illustrated in Fig. 1. This is believed to occur because the interaction length of the exciton energy transfer should be much shorter than the optical penetration length of organic semiconductors, even if the transfer is based on Förster resonance energy transfer (FRET) [19, 20], which is known to be efficient and long ranged [21]. Note that the critical distance of FRET is usually within a few nanometers, even for two-dimensional-layer energy-acceptor systems [22]. We believe that an initial distribution of excitons this narrower could be advantageous for determining the $L_{\mathrm{D}}$ of nonluminescent materials by using the photocurrent response of PV cells. Here we will demonstrate the measurement of the $L_{\mathrm{D}}$ of several Pc derivatives with different metal ions at their center (MPc; $\mathrm{M}=\mathrm{H}_{2}, \mathrm{Cu}$, and $\mathrm{Sn}$ ) by using OPV cells that exhibit interlayer excitation energy transfer. We measure MPc-thickness-dependent photocurrent responses of OPV cells with two p-type organic semiconductor layers: $\alpha$, $\omega$-diphenyl-sexithiophene (P6T) and MPc. 


\section{Experimental Details}

Figure 2a shows the chemical structures used in this study. The band gap of P6T is larger than those of the MPcs, as indicated in the figure; interlayer ETs from P6T to all MPc's are promising and have been confirmed in previous literature except for the combination of P6T and $\mathrm{H}_{2} \mathrm{Pc}$ [18]. The chemicals P6T (Sumitomo Seika Chemicals), $\mathrm{H}_{2} \mathrm{Pc}$ (Aldrich), SnPc (Aldrich), and bathocuproine (BCP, TCI) were purified before use by temperature-gradient train sublimation with Ar gas flow. We also used $\mathrm{C}_{60}$ purchased from Frontier Carbon (nanom purple SUH grade) and CuPc (organic light-emitting device grade) donated by Nippon Steel Chemicals, both without further purification. Figure $2 \mathrm{~b}$ shows the schematic of the device employed in the present study. Figure 3 shows the absorption spectra of 40-nm-thick P6T, 10-nm-thick $\mathrm{C}_{60}$, and 40-nm-thick $\mathrm{CuPc}$ layers. We prepared the devices by sequentially depositing several materials onto commercially available indium-tin-oxide (ITO) coated glass substrates, with a sheet resistance of $15 \Omega /$ square. The substrates were washed with detergent water, pure water, and 2-propanol under ultrasonication and treated with $\mathrm{O}_{2}$ plasma at $50 \mathrm{~W}$ for $5 \mathrm{~min}$. We prepared a poly(3,4-ethylenedioxythiophene):poly(styrenesulfonate) (PEDOT:PSS) layer on the substrates by spin coating a commercially available formula (Heraeus, Clevios P AI 4083). We deposited several organic layers by thermal evaporation under vacuum $\left(2.0 \times 10^{-4} \mathrm{~Pa}\right)$ at a rate of $0.6 \AA / \mathrm{s}$. Finally, the aluminum electrode was prepared on top of the organic layer stack by thermal evaporation under vacuum at a rate of $10 \AA / \mathrm{s}$. The active area of each cell was $\sim 6 \mathrm{~mm}^{2}$. We mounted the produced devices in a small chamber with a quartz window and evacuated the chamber using a rotary pump. We recorded the incident photon-current conversion efficiency (IPCE) 
spectra of the devices with a source meter (Keithley 2400) under monochromatic light irradiation from a light source (Bunko Keiki SM-25A); monochromatic light intensity for each wavelength was measured with a calibrated silicon photodiode (Hamamatsu Photonics S1337-1010BQ).

\section{Results and Discussion}

Figure 4a shows the IPCE spectra of OPV cells exhibiting the P6T and CuPc interlayer excitation energy transfer system with CuPc thicknesses varying from 7 to 25 $\mathrm{nm}$. The two peaks at $600-700 \mathrm{~nm}$ are attributed to direct optical absorption by the CuPc layer, as shown in Fig. 3. Roughly speaking, the IPCE values for CuPc do not vary as the CuPc thickness increases. However, the IPCE values decreased drastically at 400-550 nm, as shown in Fig. 4b. According to our previous report[18], the IPCE values at $400-550 \mathrm{~nm}$ are due to P6T. The incident light is absorbed by P6T, then interlayer excitation energy transfer converts $\mathrm{P} 6 \mathrm{~T}$ excitons into $\mathrm{CuPc}$ ones, and finally these $\mathrm{CuPc}$ excitons transform into charged carriers at the $\mathrm{CuPc} / \mathrm{C}_{60}$ interface. For charge separation of $\mathrm{CuPc}$ excitons, the excitons have to diffuse (migrate) from the vicinity of the $\mathrm{P} 6 \mathrm{~T} / \mathrm{CuPc}$ interface to the $\mathrm{CuPc} / \mathrm{C}_{60}$ interface. Thus, the thickness of the CuPc layer strongly influences the sensitization.

Here we would like to derive the fraction $(F)$ of excitons generated by excitation transfer for the charge separation at the $\mathrm{CuPc} / \mathrm{C}_{60}$ interface. For simplicity, we consider only the direction of thickness ( $x$-axis). The differential equation for the exciton density $(n(x, t))$ in the CuPc layer is

$$
\frac{\partial n}{\partial t}=D \frac{d^{2} n}{d x^{2}}+G(x)-\frac{n}{\tau}
$$


where $D$ and $t$ represent the diffusion coefficient and the lifetime of the exciton, respectively, and $G$ represents the generation rate function of the exciton. There are two kinds of $\mathrm{CuPc}$ excitons in the layer of our cells: light-absorption-generated excitons and excitation-transfer-generated ones. $G$ can be expressed as the sum of the two corresponding terms: the ET term $\left(G_{\mathrm{ET}}\right)$ and the light absorption one $\left(G_{\mathrm{LA}}\right)$. Thus,

$$
G(x)=G_{E T}(x)+G_{L A}(x)
$$

Here we assume that $G_{\mathrm{ET}}(x)$ is equal to the delta function $\delta(x)$. On the other hand, $G_{\mathrm{LA}}$ is assumed to be zero under blue light irradiation for measuring the IPCE at a particular wavelength because $\mathrm{Pc}$ is a blue pigment. Thus, at steady state, the differential equation for the CuPc excitons inside the layer is [23]

$$
\frac{\partial n}{\partial t}=0=D \frac{\partial^{2} n}{\partial x^{2}}-\frac{n}{\tau}
$$

The boundary conditions are $n(x=0)=n_{0}$ (where $n_{0}$ is a constant value) and $n(x=d)=$ 0 (where $d$ is the thickness of the $\mathrm{CuPc}$ layer) because it is assumed that $\mathrm{CuPc}$ excitons are fully transformed into an electron-hole pair at the $\mathrm{CuPc} / \mathrm{C}_{60}$ interface. The solution of $n(x)$ is

$$
n(x)=n_{0} \frac{\sinh \left(\frac{d-x}{L_{D}}\right)}{\sinh \left(\frac{d}{L_{D}}\right)}
$$

where $L_{D} \equiv \sqrt{D \tau}$ represents the diffusion length of the excitons. The fraction of excitons contributing to the charge separation at the $\mathrm{CuPc} / \mathrm{C}_{60}$ interface, $F$, can be expressed by $F=\frac{N_{0}-N}{N_{0}}$, where 


$$
\begin{aligned}
& N=\int_{0}^{d} n(x) d x \\
& N_{0}=\int_{0}^{\infty} n_{0} e^{-x / L_{D}} d x=n_{0} L_{D}
\end{aligned}
$$

$N$ denotes the total number of excitons resting on the CuPc layer with a thickness of $d$, while $N_{0}$ denotes the total number of excitons on the layer whose thickness is assumed to be infinite (no charge separation occurs) [23]. Finally, an analytical derivation gives the following equation for the fraction:

$$
F=\frac{\sinh \left(\frac{d}{L_{D}}\right)-\cosh \left(\frac{d}{L_{D}}\right)+1}{\sinh \left(\frac{d}{L_{D}}\right)}
$$

Figure 5 shows $F$ varying with $L_{\mathrm{D}}$ for different values of $d$. Basically, $F$ decreases almost linearly, gradually approaching zero with increases in $d$.

Here, P6T excitons are created by optical excitation, and their distribution in the layer is potentially influenced by optical interference. The effect distorts the distribution depending on $d$, the Pc layer's thickness. Therefore, optical interference can negligibly influence the analysis presented above. Indeed, the 40-nm-thick P6T layer can absorb $45 \%$ of the incident light at $440 \mathrm{~nm}$ as shown in Fig. 3. In addition, the 10-nm-thick $\mathrm{C}_{60}$ layer can absorb $\sim 10 \%$ of the incident light. Totally, $\sim 50 \%$ of the incident light can arrive at the $\mathrm{Al}$ electrode from a simple consideration. In this situation, interference does occur, albeit slightly weak. Therefore, in this study, the optical interference is assumed to be uninfluential. Note that no optical interference is influential if the $L_{\mathrm{D}}$ of P6T is greater than the thickness. However, the $L_{\mathrm{D}}$ of P6T has not been reported. 
The experimental results can be analyzed by applying the obtained IPCE values in the above derivation. However, in this study, the ET interaction distance due to the Förster mechanism should not be negligible, because the Förster critical radius for suitable combinations of excitation energy donors and acceptors is approximately a few nanometers [21]. In addition, there is a concern that the P6T layer could directly come into contact with the $\mathrm{C}_{60}$ layer when the CuPc layer is thin. Therefore, we applied the IPCE data from several to a few tens of nanometers in the following equation:

$$
\operatorname{IPCE}(d)=A \cdot F\left(d, L_{D}\right)+B
$$

where $A$ and $B$ denote the scale factor and constant value, respectively, owing to contributions to IPCE from other materials and mechanisms. For example, chargecarrier transport generated at the $\mathrm{p} / \mathrm{n}$ junction to the electrodes should be the most important mechanism in $A$. The transport is expected to be essentially dependent on $d$, because the holes generated at the junction have to travel through a Pc layer. The thicker the layer is, the more resistive the cell becomes. However, because IPCE is measured at the short-circuit condition, it is assumed that the charge-carrier transport issue does not strongly affect the results. In general, the series resistance of a photovoltaic cell, which is strongly related to charge transport, becomes more obvious around the open circuit voltage $\left(V_{\mathrm{OC}}\right)$. The fill factor, which is also related to charge transport, reduces current drastically near the $V_{\mathrm{OC}}$, not at the short-circuit condition. Therefore, in this study, $A$ is presumed to be independent of $d$. Figure $4 \mathrm{~b}$ also shows the best curve obtained by applying the data from 10 to $25 \mathrm{~nm}$. Although a large error at $d=7 \mathrm{~nm}$ was observed, the curve fits the data from 10 to $25 \mathrm{~nm}$ very well. As a result, we obtained an $L_{\mathrm{D}}$ of 4.7 $\pm 0.6 \mathrm{~nm}$. Other possible influences on this analysis include roughness at the interfaces (P6T/CuPc and $\mathrm{CuPc} / \mathrm{C} 60)$, undulating layer thickness, and interlayer mixing (e.g., 
diffusion of $\mathrm{C}_{60}$ molecules into the $\mathrm{CuPc}$ layer). We will treat such issues in future work.

Each panel in Fig. 6 shows the IPCE spectra of cells with other Pcs (SnPc and $\mathrm{H}_{2} \mathrm{Pc}$ ) of different thicknesses. As seen from each panel, the change in IPCE at the blue light region depends on the thickness of the Pc owing to light absorption by P6T, similar to the case of CuPc. Each panel in Fig. 6 shows the thickness dependence of the IPCE at $440 \mathrm{~nm}$ in each Pc. The curves in red are the best fitted results obtained from limited data ranging from $10 \mathrm{~nm}$ to maximum thickness. The $L_{\mathrm{D}} \mathrm{S}$ for the Pcs obtained are listed in Table 1. All the Pcs except SnPc have similar $L_{\mathrm{D}} \mathrm{S}$ of $\sim 5 \mathrm{~nm}$, and $L_{\mathrm{D}}$ of $\mathrm{SnPc}, 18.5 \pm$ $4.8 \mathrm{~nm}$, is much larger than those of the others. The most stable crystal structure of SnPc is triclinic [24], and those of the other Pcs, which have planar structure, are monoclinic $[25,26]$. Molecules in the triclinic SnPc crystal align themselves as J-aggregates [24], so their excitonic molecular interactions are stronger than those in other Pcs [27]. We believe this is one reason why SnPc, when compared with the others, shows a longer $L_{\mathrm{D}}$. Another reason is the difference in the electronic spin multiplicities of the excitons of the Pcs. Tin, the center atom of SnPc, is the heaviest among the center atoms of the Pcs used in this study. Owing to the heavy atom effect, a triplet exciton, which has a longer lifetime than a singlet one, can be produced. Since a longer lifetime potentially leads to a longer $L_{\mathrm{D}}$, this could also be a possible reason for the longer $L_{\mathrm{D}}$ of $\mathrm{SnPc}$.

\section{Conclusion}

We investigated the $L_{\mathrm{D}} \mathrm{S}$ of several Pcs by using cells with interlayer excitation energy transfer. The distribution of excitation-transfer-generated excitons was much narrower than that of light-absorption-generated excitons. This allowed us to analyze the exciton diffusions based on delta-function-like generation of excitons. The results of 
the analysis indicate that the planar Pcs, $\mathrm{CuPc}$ and $\mathrm{H}_{2} \mathrm{Pc}$, had shorter $L_{\mathrm{D}} \mathrm{S}(\sim 5 \mathrm{~nm})$ than those of the pyramidal-shaped $\mathrm{Pc}, \operatorname{SnPc}(18.5 \pm 4.8 \mathrm{~nm})$. If a material with a sufficiently long $L_{\mathrm{D}}$ is available as the energy donor for the excitation transfer, optical interference should have no influence on the measurement of $L_{\mathrm{D}}$ by this method. Future studies will approach this method from that viewpoint.

Acknowledgements: This work was supported by the JST PRESTO program (Photoenergy Conversion Systems and Materials for the Next Generation Solar Cells).

\section{References}

[1] F.C. Krebs, Sol. Energy Mater. Sol. Cells 93/4 (2009) 394.

[2] D. Carsten, D. Vladimir, Rep. Prog. Phys. $73 / 9$ (2010) 096401.

[3] C.J. Brabec, N.S. Sariciftci, J.C. Hummelen, Adv. Funct. Mater. 11/1 (2001) 15.

[4] M. Hiramoto, H. Fujiwara, M. Yokoyama, Appl. Phys. Lett. 58/10 (1991) 1062.

[5] B.A. Gregg, J. Sprague, M.W. Peterson, J. Phys. Chem. B 101/27 (1997) 5362.

[6] D.E. Markov, E. Amsterdam, P.W.M. Blom, A.B. Sieval, J.C. Hummelen, J. Phys. Chem. A 109/24 (2005) 5266.

[7] S.R. Scully, M.D. McGehee, J. Appl. Phys. 100/3 (2006) 034907.

[8] P.E. Shaw, A. Ruseckas, I.D.W. Samuel, Adv. Mater. 20/18 (2008) 3516.

[9] L. Lüer, H.J. Egelhaaf, D. Oelkrug, G. Cerullo, G. Lanzani, B.H. Huisman, D. de Leeuw, Org. Electron. 5/1-3 (2004) 83.

[10] D.E. Markov, P.W.M. Blom, Phys. Rev. B 74/8 (2006) 085206.

[11] L.A.A. Pettersson, L.S. Roman, O. Inganas, J. Appl. Phys. 86/1 (1999) 487.

[12] T. Stübinger, W. Brütting, J. Appl. Phys. 90/7 (2001) 3632. 
[13] P. Peumans, A. Yakimov, S.R. Forrest, J. Appl. Phys. 93/7 (2003) 3693.

[14] T. Osasa, Y. Matsui, T. Matsumura, M. Matsumura, Sol. Energy Mater. Sol. Cells 90/18-19 (2006) 3136.

[15] Y. Terao, H. Sasabe, C. Adachi, Appl. Phys. Lett. 90/10 (2007) 103515.

[16] L.-G. Yang, H.-Z. Chen, M. Wang, Thin Solid Films 516/21 (2008) 7701.

[17] S. Banerjee, A.P. Parhi, S.S.K. Iyer, S. Kumar, Appl. Phys. Lett. 94/22 (2009) 223303.

[18] M. Ichikawa, E. Suto, H.-G. Jeon, Y. Taniguchi, Org. Electron. 11/4 (2010) 700.

[19] T. Förster, Ann. Phys. 2 (1948) 55.

[20] T. Förster, Discuss. Faraday Soc. 27 (1954) 7.

[21] P.G. Wu, L. Brand, Anal. Biochem. 218/1 (1994) 1.

[22] D.R. Haynes, A. Tokmakoff, S.M. George, J. Chem. Phys. 100/3 (1994) 1968.

[23] S.M. Sze, Physics of semiconductor devices, John Wiley \& Sons, New York, 1981, p. 53.

[24] A. Yamashita, S. Matsumoto, S. Sakata, T. Hayashi, H. Kanbara, J. Phys. Chem. B 102/26 (1998) 5165.

[25] C.J. Brown, J. Chem. Soc. A (1968) 2488.

[26] S. Matsumoto, K. Matsuhama, J. Mizuguchi, Acta Crystallogr. C 55/1 (1999) 131.

[27] M. Kasha, H.R. Rawls, M.A. El-Bayoumi, Pure Appl. Chem. 11/3-4 (1965) 371. 


\section{Figure Captions}

Table 1. $L_{\mathrm{D}}$ values obtained for the Pcs

Figure 1. Schematic illustration of exciton distributions formed by ET and optical absorption in the conditional layer. Light incidences from an edge at distance $=0$. Inset: Schematic of OPV cell based on interlayer ET.

Figure 2. Structures of chemicals (a) and device (b) used in this study. Parenthetical values in panel (a) show the band gap of each material in electron volts.

Figure 3. Optical absorption spectra of P6T $(40 \mathrm{~nm}), \mathrm{C}_{60}(10 \mathrm{~nm})$, and CuPc $(40 \mathrm{~nm})$ thin films. The P6T layer was prepared on a 30-nm-thick PEDOT:PSS covered quartz substrate, but the $\mathrm{CuPc}$ and $\mathrm{C}_{60}$ layers were on a bare one.

Figure 4. (a) IPCE spectra of CuPc-based cells with different CuPc thicknesses, (b) CuPc thickness dependence of IPCE at $440 \mathrm{~nm}$, where sensitization by P6T is obvious. Data plots are presented as the average and standard deviation from two cells prepared simultaneously on the same substrate. The red line in panel (b) is the best fit curve (see text) of the data for thicknesses from 10 to $25 \mathrm{~nm}$.

Figure 5. Fraction with different $L_{\mathrm{D}} \mathrm{s}$ varying from 2 to $40 \mathrm{~nm}$ (increments of $2 \mathrm{~nm}$, shown by the black arrow) as a function of $d$. 
Figure 6. IPCE spectra of cells with two Pcs of varying thickness. (a) SnPc and (b) $\mathrm{H}_{2} \mathrm{Pc}$.

Figure 7. Thickness dependence of IPCE at $440 \mathrm{~nm}$ for cells with (a) SnPc and (b) $\mathrm{H}_{2} \mathrm{Pc}$. Data plots are presented as the average and standard deviation from two cells prepared simultaneously on the same substrate. 
Table 1. $L_{\mathrm{D}}$ values obtained for the Pcs

\begin{tabular}{llll}
\hline & $\mathrm{CuPc}$ & $\mathrm{SnPc}$ & $\mathrm{H}_{2} \mathrm{Pc}$ \\
\hline$L_{\mathrm{D}}(\mathrm{nm})$ & $4.7 \pm 0.6$ & $18.5 \pm 4.8$ & $6.5 \pm 2.1$ \\
\hline
\end{tabular}

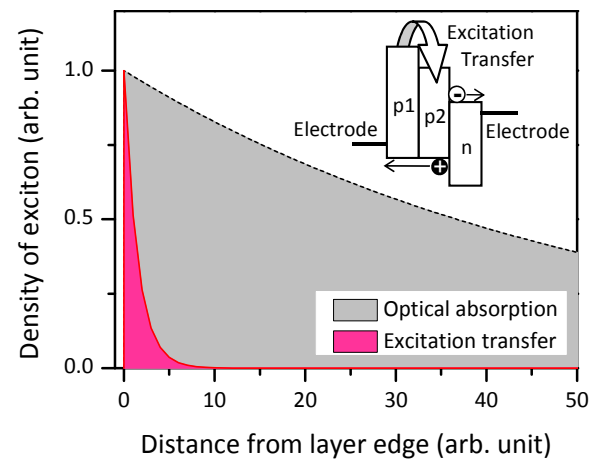

Figure 1. Schematic illustration of exciton distributions formed by ET and optical absorption in the conditional layer. Light incidences from an edge at distance $=0$. Inset: Schematic of OPV cell based on interlayer ET. 
(a)

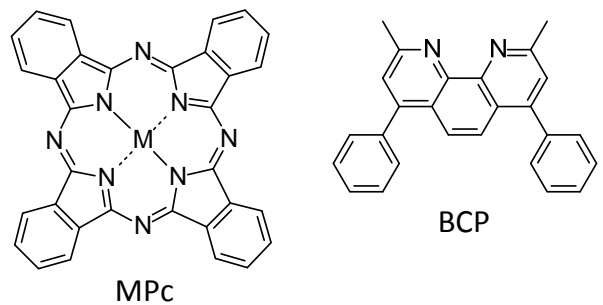

$\left(M=H_{2}(1.7), C u(1.7), S n(1.5)\right)$

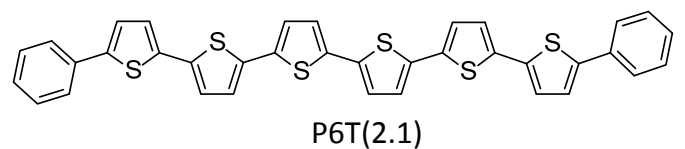

(b)

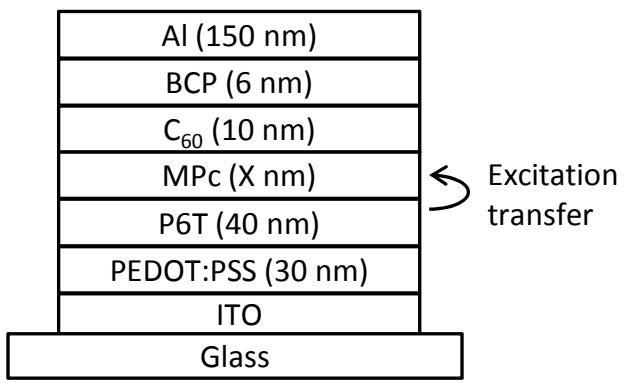

Figure 2. Structures of chemicals (a) and device (b) used in this study. Parenthetical values in panel (a) show the band gap of each material in electron volts. 


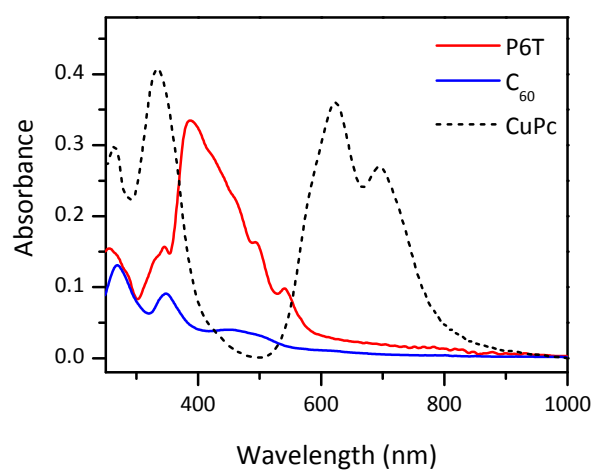

Figure 3. Optical absorption spectra of P6T $(40 \mathrm{~nm}), \mathrm{C}_{60}(10 \mathrm{~nm})$, and CuPc $(40 \mathrm{~nm})$ thin films. The P6T layer was prepared on a 30-nm-thick PEDOT:PSS covered quartz substrate, but the $\mathrm{CuPc}$ and $\mathrm{C}_{60}$ layers were on a bare one.
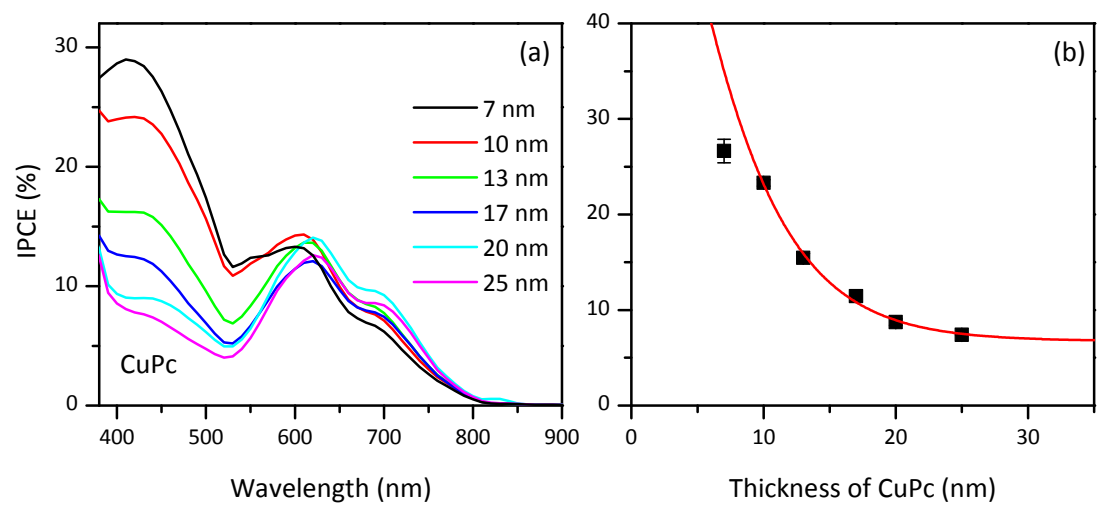

Figure 4. (a) IPCE spectra of CuPc-based cells with different CuPc thicknesses, (b) CuPc thickness dependence of IPCE at $440 \mathrm{~nm}$, where sensitization by P6T is obvious. Data plots are presented as the average and standard deviation from two cells prepared simultaneously on the same substrate. The red line in panel (b) is the best fit curve (see text) of the data for thicknesses from 10 to $25 \mathrm{~nm}$. 


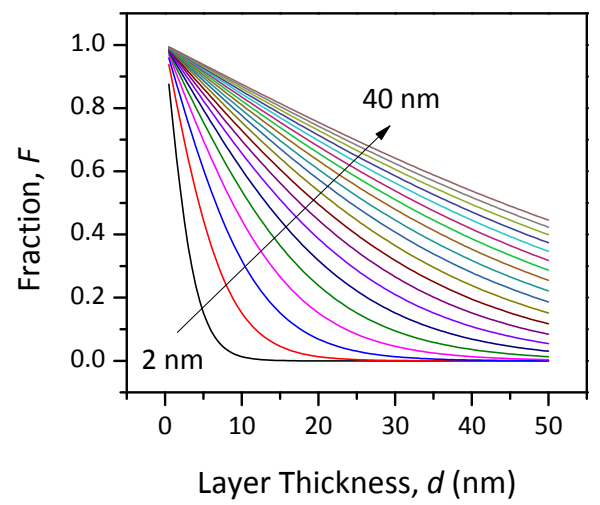

Figure 5. Fraction with different $L_{\mathrm{D}} \mathrm{s}$ varying from 2 to $40 \mathrm{~nm}$ (increments of $2 \mathrm{~nm}$, shown by the black arrow) as a function of $d$.

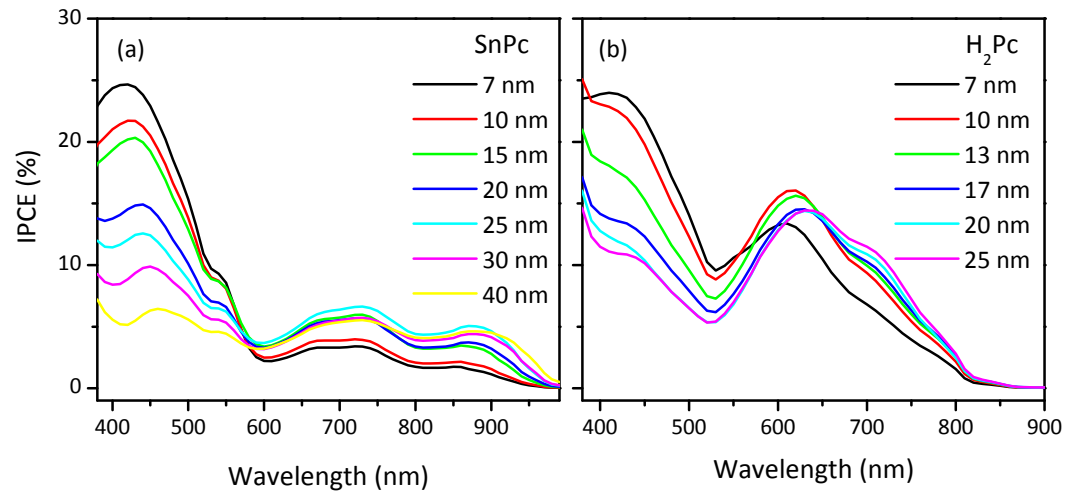

Figure 6. IPCE spectra of cells with two Pcs of varying thickness. (a) SnPc and (b) $\mathrm{H}_{2} \mathrm{Pc}$. 


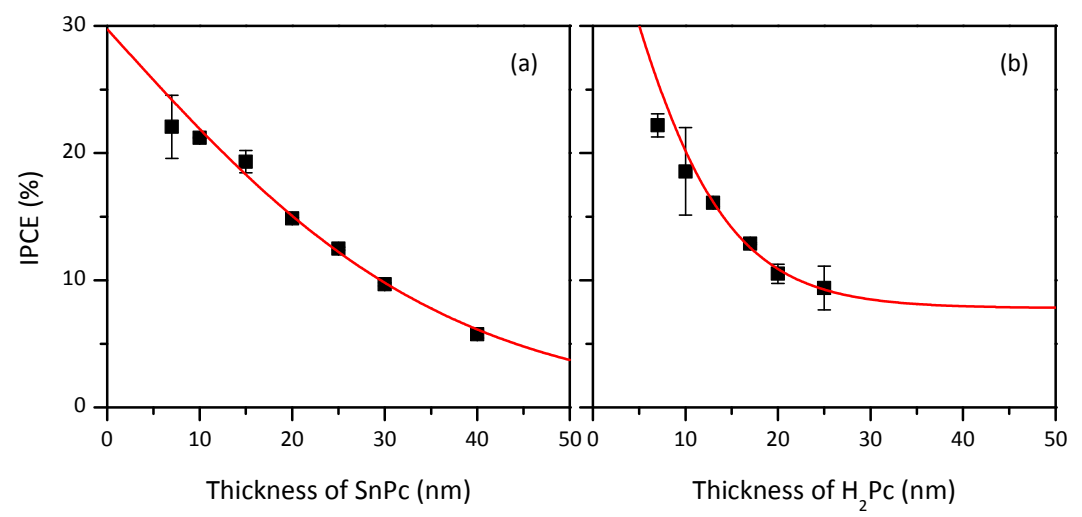

Figure 7. Thickness dependence of IPCE at $440 \mathrm{~nm}$ for cells with (a) SnPc and (b) $\mathrm{H}_{2} \mathrm{Pc}$. Data plots are presented as the average and standard deviation from two cells prepared simultaneously on the same substrate. 